\title{
Supernova remnants and their progenitors
}

\author{
John R. Dickel
}

Department of Physics and Astronomy, University of New Mexico, 800 Yale Blvd. NE, Albuquerque, NM 87131, USA

email: johnd@phys.unm.edu

Abstract. Young supernova remnants (SNRs) show obvious differences that can be related to characteristics of the progenitors and supernova types as tabulated in Fig. 1. Questions remain.

Keywords. ISM: supernova remnants, supernovae: general, acceleration of particles

Questions include: why do some Type Ia supernova remnants show a definite gap between the leading-shock rim and the main shell (e.g., Tycho), while others show a single continuous shell out to the sharp rim (SN 1006)?

Why do some SNe leave only a cooling neutron star with no pulsar wind nebula (PWN) (Cas A), or erratic point X-ray sources (RCW 103), while others have pulsars? Is it the magnetic field, spin rate or what?

What physical conditions can discriminate between mixed morpology remnants with internal thermal X-rays (W 44) and those with more standard shells (Vela)? Is it just the surrounding medium?

The composite SNR G11.2-0.3 has been identified with SN 386. It contains a central pulsar with a period of $65 \mathrm{~ms}$ and a calculated spin-down age about three times longer than the age since its explosion. In contrast, the Crab Nebula, an extended pulsar wind nebula with no apparent shell, has a close match between the two ages. Is the presence or absence of a shell related to differences in the moment of inertia of the neutron star?

Why do some composite SNRs (MSH 15-56) show a radio pulsar wind nebula somewhat offset from the center, and then a point X-ray source with an apparent X-ray PWN out near the shell and not necessarily aligned with the radio PWN?

\begin{tabular}{|c|c|c|c|c|}
\hline & $\begin{array}{c}\text { Low Mass } \\
\text { Type Ia }\end{array}$ & \multicolumn{3}{|c|}{$\begin{array}{c}\text { High Mass } \\
\text { Type Ib or II }\end{array}$} \\
\hline $\begin{array}{c}\text { Examples } \\
\text { (approximate } \\
\text { explosion date } \\
\text { if known) }\end{array}$ & $\begin{array}{c}\text { Kepler (1604)? } \\
\text { Tycho (1572) } \\
\text { AD1006 (1006) } \\
\text { 0519-690 (350-1500) } \\
\text { SN1885 in M31 (1885) }\end{array}$ & $\begin{array}{c}\text { Cas A ( 1680) } \\
\text { RCW103 }(\sim 0) \\
\text { E0102-723 } \\
\text { Puppis A }\end{array}$ & $\begin{array}{l}\text { 3C58(1181) } \\
\text { Crab(1054) }\end{array}$ & \begin{tabular}{|} 
G11.2-0.3(386) \\
W44 Vela \\
0540-693 ( 0) \\
N157B \\
IC443
\end{tabular} \\
\hline Morphology & $\begin{array}{c}\text { Reasonably round } \\
\text { shells }\end{array}$ & $\begin{array}{c}\text { Broken up shells, } \\
\text { shrapnel }\end{array}$ & $\begin{array}{l}\text { Only pulsar } \\
\text { wind nebula }\end{array}$ & \begin{tabular}{|c|} 
Composite \\
PWN plus shell
\end{tabular} \\
\hline Compact core & -- & $\begin{array}{l}\text { Cooling neutron star, no } \\
\text { pulsar wind nebula }\end{array}$ & \multicolumn{2}{|c|}{$\begin{array}{c}\text { Pulsar plus pulsar wind } \\
\text { nebula (PWN) }\end{array}$} \\
\hline $\begin{array}{r}\text { Spectral results } \\
\text { optical } \\
\text { X-ray }\end{array}$ & $\begin{array}{l}\text { Balmer dominated, N,S } \\
\text { Thermal Fe, Si, S }\end{array}$ & $\begin{array}{c}\text { O (FMKs), N (QSFs) } \\
\text { Fe, metals }\end{array}$ & \multicolumn{2}{|c|}{$\begin{array}{c}\text { Ni, } \mathbf{N}, \mathbf{S} \\
\text { Power law (synchrotron) in } \\
\text { PWN }\end{array}$} \\
\hline $\begin{array}{l}\text { Pre point-blast } \\
\text { expansion }\end{array}$ & $\begin{array}{l}\text { Near point blast } \\
\quad R \propto t^{0.5}\end{array}$ & $\begin{array}{l}\text { Near free expansion } \\
\qquad R \propto t^{0.9}\end{array}$ & \multicolumn{2}{|r|}{--} \\
\hline Emvironment & $\begin{array}{c}\text { Isolated object but } \\
\text { often complex } \\
\text { circumstellar medium }\end{array}$ & Pre-explosion mass loss & \multicolumn{2}{|c|}{ Pre-explosion mass loss } \\
\hline
\end{tabular}

Figure 1. Properties of young SNRs 